\title{
Precoded Cluster Hopping for Multibeam GEO Satellite Communication Systems
}

\author{
Eva Lagunas *, Mirza Golam Kibria, Hayder Al-Hraishawi, Nicola Maturo and \\ Symeon Chatzinotas
}

Interdisciplinary Centre for Security, Reliability and Trust (SnT), University of Luxembourg, Luxembourg, Luxembourg

Beam hopping $(\mathrm{BH})$ and precoding are two trending technologies for high-throughput satellite (HTS) systems. While BH enables the flexible adaptation of the offered capacity to the heterogeneous demand, precoding aims at boosting the spectral efficiency. In this study, we consider an HTS system that employs $\mathrm{BH}$ in conjunction with precoding in an attempt to bring the benefits of both in one. In particular, we propose the concept of cluster hopping $(\mathrm{CH})$, where a set of adjacent beams are simultaneously illuminated with the same frequency resource. On this line, we propose an efficient time-space illumination pattern design, where we determine the set of clusters that shall be illuminated simultaneously at each hopping event along with the dwelling time. The $\mathrm{CH}$ time-space illumination pattern design formulation is shown to be theoretically intractable due to the combinatorial nature of the problem and the impact of the actual illumination design on the resulting interference. For this, we make some design decisions on the beam-cluster design that open the door to a less complex still well-performing solution. Supporting results based on numerical simulations are provided which validate the effectiveness of the proposed $\mathrm{CH}$ concept and a time-space illumination pattern design with respect to benchmark schemes.

Keywords: satellite communications, multibeam satellite, beam hopping, precoding, demand-matching

\section{INTRODUCTION}

The first generation of broadband multibeam satellites was launched in the 2000s, with the main objective to deliver internet services to people who had no access to faster forms of internet connectivity (ViaSat Inc., 2018). Driven by the success of the first generation of broadband satellites, new advanced satellite systems were set up during the 2010s with spot beams. Viasat-1 is a clear example of such next generation of satellites, which is able to serve 72 spot beams and reach a total capacity of $140 \mathrm{Gbps}$. Clearly, these accomplishments established the birth of the so-called generation of high-throughput satellite (HTS) systems (Cola et al., 2015). While wireline and wireless terrestrial broadband service lack the ability to leap across continents, oceans, and difficultto-access areas, the inherent large coverage footprint of satellite communication networks make them the most suitable solution to expand networks over the world. Therefore, satellites can complement the terrestrial networks and offer important socioeconomic benefits, while increasing the satellite competitiveness.

From frequency/bandwidth to power allocation and coverage, the forthcoming generation of commercial satellite communication payloads offer enhanced flexibility to dynamically satisfy the customers' demands (Kisseleff et al., 2020; NetWorld 2020, 2019). Such reconfigurable satellite systems are clamored by operators and manufacturers to be one of the most groundbreaking 
evolutions of satellite communications with an impact on lowering mission costs and enabling satellite systems to become more agile and responsive to market needs (SES, 2020; AIRBUS, 2021). These future satellite architectures are expected to offer terabit per second in-orbit capacity when and where needed. Such throughput enhancements can only be achieved by pushing forward the multibeam architecture with a reduced beam size, taking advantage of frequency reuse and reconfiguring the satellite capacity according to the heterogeneous traffic demands.

In response to the combination of ever-growing data demand with the inherent satellite spectrum scarcity (Kodheli et al., 2020), an intelligent allocation of satellite resources considering the new degrees of flexibility shall be conceived, particularly considering both the actual users' position as well as their traffic demand. This study focuses on two of the most promising disruptive techniques to tackle these specific challenges: linear precoding and timeflexible beam hopping.

\subsection{Linear Precoded for Satellite Systems}

While conventional satellite systems are designed to operate using an interference avoidance approach through a proper reuse of the available spectrum among beams, more recent paradigms have been proposed and studied which go in the opposite direction through the management and the exploitation of the interference among beams. The objective is clearly to maximize the use of the user link available spectrum (in terms of spectral efficiency), which represents a limited resource of the system. In this context, Vazquez et al. (2016) summarize multiuser multiple input single output (MU-MISO) digital signal processing techniques, such as linear precoding, that can be applied in the user link of a multibeam satellite system operating in full frequency reuse. While the concept of MU-MISO in satellite networks have been mostly theoretical, an actual live-based demonstration supported by the European Space Agency (ESA) has been carried out in ESA project LiveSatPreDem (2020), validating the feasibility of such technique considering the recently amended DVB-S2X specifications to support it. It is worth to remark that precoding is embedded at the gateway, thus keeping the complexity of the payload and user terminal (UT) infrastructure low.

In general, one of the main challenges faced by HTS systems (particularly for precoded systems) is the feeder link congestion, that is, the congestion on the bidirectional communication link between the gateway and the satellite. The increase in the capacity of the user link requires a corresponding increase in the capacity of the feeder link, which is currently limited by few $\mathrm{GHz}$ of available bandwidth (Kyrgiazos et al., 2014). In principle, the exploitation of higher frequency bands (e.g., Q/V) by this wireless link could address this issue. However, often this approach is not feasible in practice due to weather impairments at high frequencies (Zhang et al., 2017). A common alternative is the deployment of multiple gateways, where each gateway conveys the signals to be transmitted to a cluster of spot beams. This concept of beam-clustering would be relevant to this study and will be addressed in the next section.

\subsection{Time-Flexible Beam Hopping}

Beam hopping $(\mathrm{BH})$ was originally proposed to deal with large multibeam coverage areas, by focusing the satellite resources to certain subset of beams, which is active for some portion of time, dwelling just long enough to satisfy the requested demands (Freedman et al., 2015). In doing so, BH is able to increase useable capacity and reduce unmet traffic demands, particularly in the presence of heterogeneous traffic demand.

The conventional $\mathrm{BH}$ illumination pattern is illustrated in Figure 1A, where the active spot beams are designed to have a border area formed by inactive beams such that a degree of isolation exists between each active beam. Note that the set of illuminated beams changes in each time slot based on a time-space transmission pattern that is periodically repeated. The time axis is divided in windows of duration $T_{H}$, which repeat following a regular pattern. Each $\mathrm{BH}$ window is segmented in $N_{s}$ time slots and in each time slot a different set of beams is illuminated. By modulating the period and duration that each of the beams is illuminated, different offered capacity values can be achieved in different beams.

The $\mathrm{BH}$ procedure on the one hand allows higher frequency reuse schemes by placing inactive beams as barriers for the cochannel interference, and on the other hand allows the use of a reduced number of onboard power amplifiers, with a consequent reduction of payload mass. $\mathrm{BH}$ benefits have been well demonstrated, for example, ESA project BEAMHOP (2016), and the satellite standard DVB-S2X has recently included guidelines to enable beam hopping operation.

On the downside, we noticed that in certain scenarios where more than one adjacent beam is requesting high demand, the performance of $\mathrm{BH}$ is affected by the limitation of not being able to simultaneously activate neighboring beams with the same spectrum resource. The latter motivates the contribution of this study.

In summary, $\mathrm{BH}$ provides the means to flexibly adapt the offered capacity to the time and geographic variations of the traffic demands, while precoding exploits the multiplexing feature enabled by the use of multiple antenna feeds at the transmitter side to boost the spectral efficiency. These two effective strategies can create unique opportunities if they are properly combined.

\subsection{Contribution: Precoded Cluster Hopping}

The contributions of this study are summarized as follows:

1) Cluster hopping concept: we propose the novel cluster hopping $(\mathrm{CH})$ concept as a natural combination of $\mathrm{BH}$ with precoding. In $\mathrm{CH}$, multiple set of adjacent beams are illuminated at the same time with the same frequency resource. We define a cluster as the set of adjacent active beams that are served by a single gateway so that the whole coverage area can be served through multiple clusters/ gateways. An example of the proposed $\mathrm{CH}$ is shown in Figures 1B,C, which requires the use of precoding to deal with the resulting interference as no separation line of inactive beams is considered within a beam cluster. $\mathrm{CH}$ was first introduced by the authors in Kibria et al. (2019). Herein, 

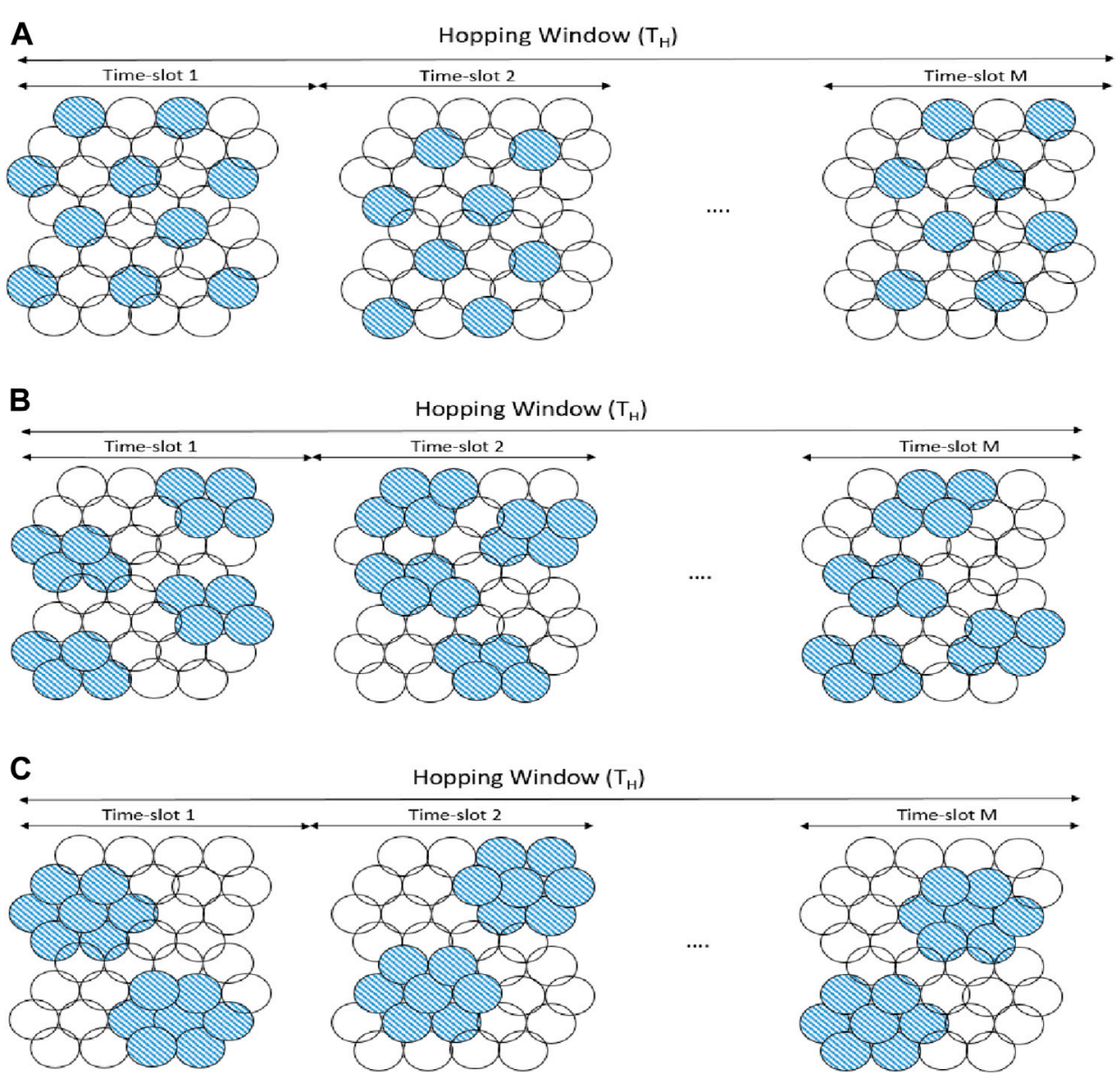

FIGURE 1 | Beam hopping illumination pattern: (A) conventional beam hopping; (B) proposed cluster hopping with four-beam clusters; and (C) proposed cluster hopping with seven-beam clusters.

we expand Kibria et al. (2019) with more technical details, expanding the numerical results.

2) Illumination pattern design: the illumination pattern design for conventional $\mathrm{BH}$ systems has been studied in AlegreGodoy et al. (2012), Angeletti et al. (2012), Anzalchi et al. (2010), Cocco et al. (2018), and Lei et al. (2020). While AlegreGodoy et al. (2012), and Anzalchi et al. (2010) focused on heuristic iterative suboptimal algorithms, Angeletti et al. (2012) and Cocco et al. (2018) considered genetic and simulated annealing algorithms, respectively, targeting global optimal solutions at the expenses of increased computational complexity. Finally, Lei et al. (2020) proposed to integrate deep learning into the optimization procedure in order to accelerate the optimization procedure. Herein, we propose an illumination pattern design for $\mathrm{CH}$ (and therefore considering precoding the corresponding clusters) under a fair beam demand satisfaction objective. In particular, we formulate the illumination pattern design as a max-min of the offered vs. demanded capacity subject to a set of practical constraints. The presence of binary assignment variable as well as nonlinearity caused by the interference as a function of such binary assignment variable makes the problem non-convex and difficult to solve. To tackle this, we propose to limit the clustering to specific forms that allow us to 1) simplify the relationship between a specific beam illumination instance and the resulting interference and 2) reduce the search space of the feasible solutions and, therefore, obtain a low-complex solution. Although optimality cannot be guaranteed, this solution is shown to reach satisfactory results with affordable complexity.

3) Numerical evaluation: finally, we present supporting results based on numerical simulations using a software tool (SnT University of Luxembourg, 2020). We evaluate the beam demand satisfaction under different beam-clustering designs and different number of simultaneously activated beams, for different demand instances. We also compare the proposed $\mathrm{CH}$ with respect to the conventional $\mathrm{BH}$ technique and with respect to Ginesi et al. (2017). The latter represents a preliminary study carried out by ESA, where precoding was first combined with $\mathrm{BH}$ and a pragmatic, iterative, and heuristic approach was proposed for the illumination pattern design. 
The rest of this article is organized as follows. Section 2 introduces the system model and relevant nomenclatures. Section 3 presents the proposed cluster hopping concept considered in this study and addresses the illumination pattern design. Supporting simulation results are presented in Section 4, and finally, concluding remarks as well as future research directions are provided in Section 6.

\section{SYSTEM MODEL}

Let us consider a high-throughput multi-beam satellite system with a total of $N_{b}$ beams, from which only a subset of $Q$ beams, $Q<N_{b}$, can be simultaneously activated at a particular time instance. We define the illumination ratio as $Q / N_{b}$, for example, a $1 / 4$ illumination ratio means that $25 \%$ of the total number of beams is illuminated. We assume that the beams that are illuminated employ full-frequency reuse, meaning that all of them operate over the same spectrum $B_{\mathrm{w}}$. For the sake of clarity, the feeder links (connection between gateways and satellite) is considered ideal, that is, noiseless and without channel impairments.

In this study, we use the following terminologies:

- Cluster: a group of adjacent beams simultaneously illuminated with the same spectrum $B_{\mathrm{w}}$. To cope with the resulting interference, clusters are precoded.

- Snapshot: a particular arrangement of illuminated and nonilluminated clusters. For illustration purposes, Figures 1B,C show three and three snapshots, respectively. There can be as many as $2^{N_{C}}$ possible snapshots, $N_{c}$ being the number of considered clusters. Of course, not all snapshots are valid in the sense that only a given number of nonadjacent clusters can be illuminated simultaneously because of payload limitations, that is, the illumination ratio.

- Time slot: a time slot or time instance defines the time granularity of the hopping operation, that is, the minimum illumination period for a selected snapshot. The hopping window, $T_{H}$, is equally divided into $N_{s}$ time slots. Therefore, $T_{H}=N_{s} \times T_{s}$, where $T_{s}$ denotes the duration of the time slot.

- Hopping window: as anticipated, the hopping window consists of $N_{s}$ time slots and has a total duration of $T_{H}$. It also represents the maximal time period allowed to provide service to all the users in the coverage area.

Let us focus on a particular snapshot and on a particular cluster within that snapshot. The signal vector received by the $N_{c}$ active beams within the $i$ th cluster is denoted as $\mathbf{y}_{i} \in \mathbb{C}^{N_{c} \times 1}$ and further expressed as:

$$
\mathbf{y}_{i}=\mathbf{H}_{i} \mathbf{x}+\mathbf{n}_{i},
$$

where $\mathbf{x} \in \mathbb{C}^{\mathrm{Q} \times 1}$ denotes the transmitted symbols with $\mathbb{E}\left[\mathbf{x x}^{H}\right]=$ $\mathbf{I}_{Q}$ and $\mathbf{H}_{i} \in \mathbb{C}^{N_{c} \times Q}$ refers to the channel matrix of cluster $i$, which includes the components of all active beams and is assumed to be perfectly known at the transmitter, and $\mathbf{n}_{i} \in \mathbb{C}^{N_{c} \times 1}$ denotes the additive Gaussian zero-mean unit-variance noise, that is, $\mathbb{E}\left[\mathbf{n}_{i} \mathbf{n}_{i}^{H}\right]=\mathbf{I}_{N_{c}}$.

For the sake of clarity, we drop the cluster subindex $i$ throughout the following, which applies to any cluster. The entry at the $k$ th row and $q$ th column of the downlink channel matrix $\mathbf{H}$ in (1) between the multibeam satellite and the $N_{c}$ beams of the cluster is modeled as:

$$
[\mathbf{H}]_{k, q}=\frac{\sqrt{G_{R x} G_{k, q}}}{4 \pi \frac{d_{k}}{\lambda} \sqrt{\kappa T_{R x} B_{\mathrm{w}}}}
$$

where

- $G_{R x}$ is the receiver antenna gain (assumed to be the same for all UT for simplicity),

- $G_{k, q}$ is the gain from the $q$ th beam seen at the $k$ th beam,

- $d_{k}$ is the distance between the satellite and $k$ th beam,

- $\lambda$ denotes the wavelength,

- $\kappa$ denotes the Boltzmann constant,

- $T_{R x}$ is the clear sky noise temperature of the receiver.

Consequently, the received signal of the $k$ th beam can be written as:

$$
y_{k}=\underbrace{\mathbf{h}_{k}^{T} x_{k}}_{\text {desired }}+\underbrace{\sum_{\substack{j \in \mathcal{C} \\ j \neq k}} \mathbf{h}_{k}^{T} x_{i}}_{\text {intra-cluster interf. }}+\underbrace{\sum_{u \notin \mathcal{C}} \mathbf{h}_{k}^{T} x_{u}}_{\text {inter-cluster interf. }}+n_{k},
$$

where $\mathbf{h}_{k}^{T}$ denotes the $k$ th row of matrix $\mathbf{H}$, and we distinguish two types of interference: (i) intra-cluster interference, with $\mathcal{C}$ denoting the set of beams belonging to the same cluster as beam $k$ and (ii) inter-cluster interference, which considers all the transmission signals not intended to cluster $\mathcal{C}$.

\section{CLUSTER HOPPING DESIGN}

Both interference components in (3) can be mitigated by considering precoding over all $Q$ active beams. Although this is the best approach in terms of achievable capacity, its implementation is limited by the feeder link congestion. For such number of active beams, multiple and coordinated gateway stations are required, which is considered unlikely in practice due to the synchronization accuracy needed for coordinated precoding (Arapoglou et al., 2016).

Therefore, our first design decision is to mitigate the intracluster interference only by precoding clusters independently. Regarding the inter-cluster interference, its effect will be minimized by considering avoiding adjacent clusters to be simultaneously activated. These two assumptions, shall allow us to 1) upload the signals using multiple noncooperative gateways, 2) pursue a design under the assumption of negligible inter-cluster interference, and 3) reduce the number of possible snapshots and, thus, the search space of the cluster hopping design. 
Taking into account the aforementioned assumptions, the offered capacity to beam $k$ belonging to cluster $\mathcal{C}$ can be expressed as:

$$
c_{k}=B_{\mathrm{w}} \mathrm{f}_{D V B}\left(\frac{P_{\text {beam }}\left|\mathbf{h}_{k}^{H} \mathbf{w}_{k}\right|^{2}}{\sum_{\substack{j \in \mathcal{C} \\ j \neq k}} P_{\text {beam }}\left|\mathbf{h}_{k}^{H} \mathbf{w}_{j}\right|^{2}+1}\right)[\text { bits/sec }],
$$

where $\mathrm{f}_{D V B}$ denotes the signal-to-interference and noise ratio (SINR) vs. the spectral efficiency (SE) mapping function according to the adaptive coding and modulation (ACM) scheme considered by the digital video broadcasting (DVB) standard (DVB-S2X, 2014). The transmit power per beam is assumed to be fixed and equally distributed across beams and it is denoted as $P_{\text {beam }}$. It is out of the scope of this study to optimize the transmit power.

As a consequence, the cluster capacity can be obtain by adding all the capacity of the beams belonging to that cluster: $C_{i}=\sum_{k \in \mathcal{C}_{i}} c_{k}$, where we have again reintroduced the cluster subindex $i$.

\subsection{Objective}

The objective is to obtain the optimal illumination pattern, that is, set of snapshots and their dwelling time, such that the demands of the beams/clusters are fairly satisfied. In other words, the optimal illumination pattern would be such that achieves $c_{i} \approx d_{i}, i=1, \ldots$, $N_{b}, C_{i} \approx D_{i}$, and $i=1, \ldots, N_{c}$, where $d_{i}$ and $D_{i}$ denote the demand of $i$ th beam and $i$ th cluster, respectively. Note that this study focuses on the demand-matching at the beam level. The task to distribute the beam capacity to the different end-users of that beam is known as user scheduling (Guidotti and Vanelli-Coralli, 2020; Honnaiah et al., 2021).

\subsection{Proposed Illumination Design}

Let us define our design variable with a set of binary vectors $\mathbf{x}_{t}$ of dimension $N_{c} \times 1$, with components $x_{t}$ [i] being equal to one when cluster $i$ is active at the time slot $t$.

Since the optimization of the illumination design is performed at the hopping window level, we scale down the cluster demand at the hopping-window level as $\hat{D}_{i}=T_{H} D_{i}$ [bits/hopping window], and the offered cluster capacity at time slot level as $\hat{C}_{i}=T_{s} C_{i}$ [bits/time-slot]. With these definitions, we can state that the actual offered capacity at the hopping window level can be computed as $\hat{R}_{i}=\sum_{t=1}^{N_{s}} x_{t}[i] \hat{C}_{i} \quad$ [bits/hopping window], where the cluster offered capacity $\hat{C}_{i}$ can be easily precomputed and stored.

As discussed, without making any assumption on the snapshot design, the number of possible binary arrangements in $\mathbf{x}_{t}$ is $2^{N_{c}}$, which might become untractable for realistic multibeam patterns. However, not all are valid snapshots for our problem as we have a couple of constraints, namely, maximum number of active beams per time slot (i.e., $\sum_{i=1}^{N_{c}} x_{t}[i]=Q^{\prime}$ ), $Q^{\prime}$ denoting the number of active clusters, and activation of adjacent clusters shall be avoided. The latter constraint can be expressed as:

$$
\mathbf{x}_{t}^{T} \mathbf{A} \mathbf{x}_{t}=0
$$

where matrix $\mathbf{A} \in\{0,1\}^{N_{c} \times N_{c}}$ represents the binary adjacency matrix of the clusters. It is a square symmetric matrix, that is, $\mathbf{A}_{i, j}=\mathbf{A}_{j, i}$ and $\mathbf{A}_{i, j}=1$ when cluster $i$ is adjacent to cluster $j$.

With all these in mind, the proposed cluster illumination pattern design is formulated in the following equation:

$$
\begin{array}{ll}
\max \min _{\left\{\mathbf{x}_{t}, t=1, \ldots, N_{s}\right\}} & \left(\frac{\hat{R}_{1}}{\hat{D}_{1}}, \ldots, \frac{\hat{R}_{N_{c}}}{\hat{D}_{N_{c}}}\right) \\
\text { s.t. } & \sum_{i=1}^{N_{c}} x_{t}[i]=Q^{\prime}, \\
& \mathbf{x}_{t}^{T} \mathbf{A} \mathbf{x}_{t}=0, \mathrm{t}=1, \ldots, N_{s} \\
& x_{t}[i] \text { binary, } \mathrm{t}=1, \ldots, N_{s} \mathrm{i}=1, \ldots, N_{c}
\end{array}
$$

We can simplify the max-min optimization problem in (6) by turning it into a maximization problem with the help of an additional slack variable $\gamma$ along with a new constraint $\frac{\hat{R}_{1}}{\hat{D}_{1}} \geq \gamma \triangleq \hat{R}_{1} \geq \hat{D}_{1} \gamma$ :

$$
\begin{array}{ll}
\max _{\left\{\mathbf{x}_{t}, t=1, \ldots, N_{s}\right\}} & \gamma \\
\text { s.t. } & \sum_{i=1}^{N_{c}} x_{t}[i]=Q^{\prime}, \\
& \mathbf{x}_{t}^{T} \mathbf{A x}_{t}=0, \mathrm{~m}=1, \ldots, N_{s} \\
& x_{t}[i] \text { binary, } \mathrm{t}=1, \ldots, N_{s} \mathrm{i}=1, \ldots, N_{c} \\
& \hat{R}_{i} \geq \hat{D}_{i} \gamma, \mathrm{i}=1, \ldots, N_{c}
\end{array}
$$

One can observe that problem (7) is a linear programming (LP) problem involving a binary assignment variable. Although the inherent combinatorial problem remains, with the proposed constraints and a careful beam-clustering design, one can reduce significantly the search space. The beam-clustering aspects are discussed in the following section, while some numbers about the search space of problem (7) are provided in Section 4.

For solving (7), in this study, we rely on the optimization software Gurobi (GUROBI, 2021), which is convenient to solve mixed integer linear programming (MILP) problems such as the one in (7).

\subsection{Clustering Definition}

The offered capacity per cluster, that is, $\hat{R}_{i}$, strongly depends on the cluster shape and size. Deriving optimal clustering optimization would require an exhaustive search over all possible combinations of clustering options, including an irregular cluster size and overlapping clusters, rendering a huge search space. Moreover, the cluster definition also impacts on the complexity of the system as the number of possible snapshots is a function of the number of clusters. For example, a cluster with a small size will yield to a bigger search space for the problem in (7), while clusters with a big size will reduce the search space but provide less flexibility in the $\mathrm{CH}$ operation. To keep the complexity of (7) within tractable limits, we opt to have compact-shaped, nonoverlapping, and equal size cluster due to the following reasons: 


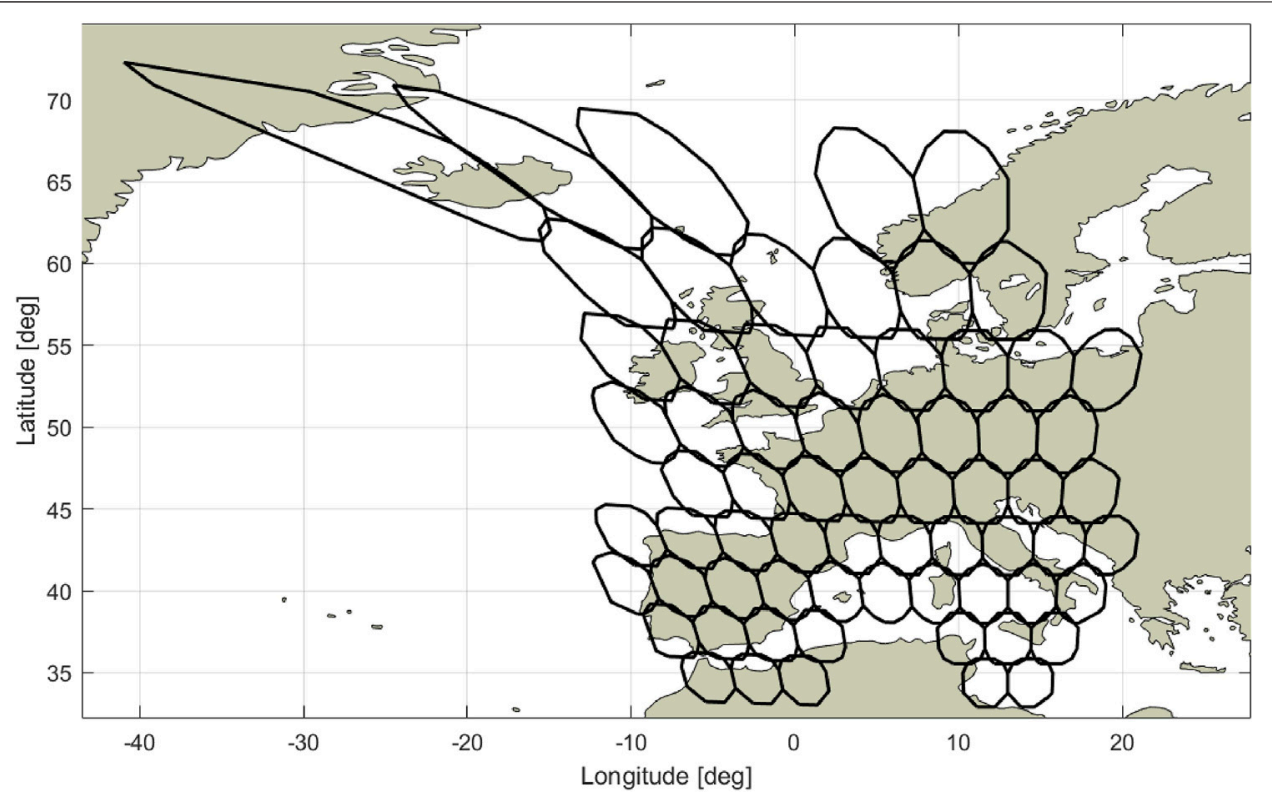

FIGURE 2 | Considered beam pattern with $N_{b}=67$ beams.

\section{TABLE 1 | Simulation parameters.}

Satellite longitude

Satellite height

Number of beams, $N_{b}$

Beam radiation pattern

Max. beam radiation pattern gain

Downlink carrier frequency

Satellite total power, $P_{\text {total }}$

User link bandwidth

Roll-off factor

Effective user link bandwidth, $B_{\mathrm{w}}$

Roll-off factor

Illumination ratio, $\left(Q / N_{b}\right)$

Duration of a time slot, $T_{S}$

Hopping window, $T_{H}$

User terminal antenna gain, $G_{R x}$

Noise power, $\left(\kappa T_{R \times} B_{\mathrm{w}}\right)$

- In the case of overlapping clusters, there will be a very large number of possible clusters making a huge search space for the proposed problem.

- Compact-shaped clusters are preferred vs. linear- or quasilinear-shaped clusters in order to exploit the precoding benefits.

- The size of the clusters, as discussed before, brings a complexity-performance trade-off. Different cluster sizes will be evaluated in Section 4.

\section{SIMULATION RESULTS}

The simulation setup for evaluating the performance of the proposed precoded $\mathrm{CH}$ in an HTS system is as follows. The 67-beam GEO satellite beam pattern shown in Figure 2 is considered. The pattern has been generously provided by ESA in the context of the project FlexPreDem (ESA Project FlexPreDem, 2020). The transmit power per beam $P_{\text {beam }}$ is a function of the illumination ratio as it is calculated as $P_{\text {beam }}=P_{\text {total }} / Q$. In other words, the total power $P_{\text {total }}$ is equally distributed across the active beams. The rest of the simulation parameters are provided in Table $\mathbf{1}$.

First of all, we provide some numbers in terms of the complexity scalability with the clustering definition. As shown in Table 2, assuming a cluster size equal to six beams for all clusters will result in a total of 21,211 clusters if no further assumptions are made. On the other hand, assuming a cluster size equal to four beams for all clusters will result in a total of 1,675 clusters. However, if we make the assumptions proposed in Section 3.3, these numbers can be reduced to 17 and 11 , respectively, resulting in a more manageable number. As a consequence, we evaluate the performance of the $\mathrm{CH}$ concept under these later clustering options, both shown in Figure 3. The actual complexity of problem (7) is dictated by the number of snapshots resulting from the combination of the clustering definition and the illumination ratio. In other words, the binary combinations within $\mathbf{x}_{t}$ are constrained by the number of clusters that can be simultaneously activated $\left(Q^{\prime}\right)$ and the adjacent cluster avoidance. Considering these constraint, the number of snapshots $N_{p}$ for different illumination ratios is given in Table 2. As expected, higher illumination ratios allow activating higher number of clusters per snapshot, therefore, resulting in higher number of possible snapshots. Still, the numbers shown in Table $\mathbf{2}$ are tractable allowing to final a solution to problem (7) in a matter of seconds with conventional personal computers.

The proposed precoded $\mathrm{CH}$ scheme is evaluated in terms of unmet capacity and unused capacity. Both are figures of merits 
TABLE 2 | Clustering impact on the combinatorial problem complexity.

\begin{tabular}{lcc}
\hline Size of cluster & $\begin{array}{c}\text { Number of clusters } \\
\text { (compact, non-compact, overlapping, } \\
\text { nonoverlapping) }\end{array}$ & $\begin{array}{c}\text { Number of clusters } \\
\text { (compact, overlapping, nonoverlapping) }\end{array}$ \\
\hline (compact, nonoverlapping) & 21,211 & 483 \\
6 Beams & 1,675 & 132 \\
\hline
\end{tabular}

Assuming compact and nonoverlapping clusters

Size of Cluster Number of snapshots (Illum. Ratio 1/4) Number of snapshots (Illum. Ratio 1/6) Number of snapshots (IIlum. Ratio 1/8)
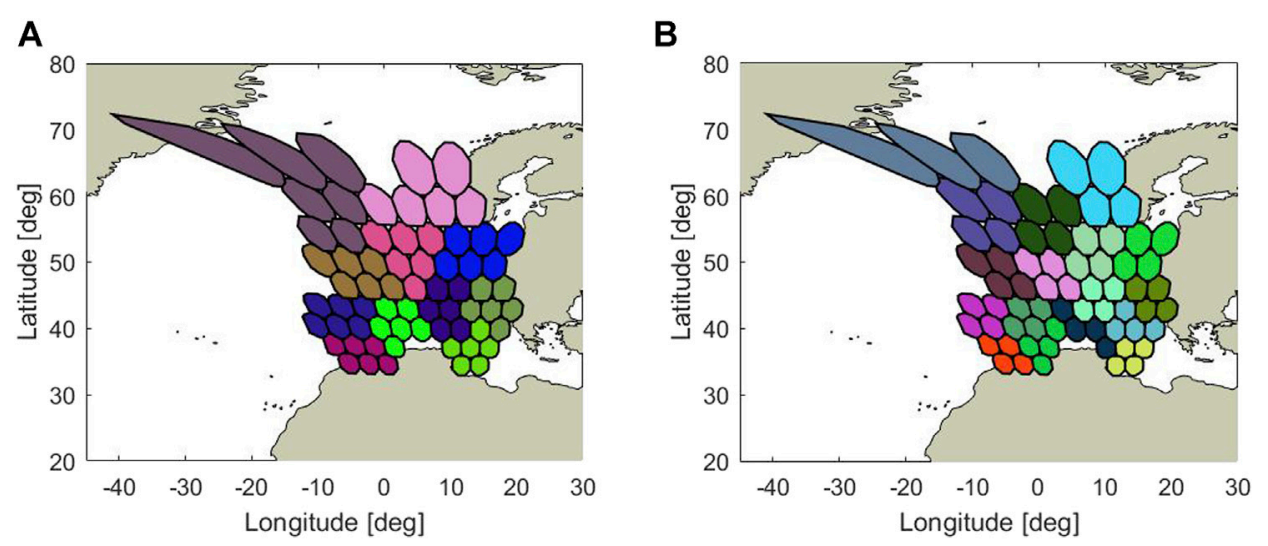

FIGURE 3 | Considered beam-clustering options: (A) 11 clusters of six beams/cluster; (B) 17 clusters with four beams/cluster.
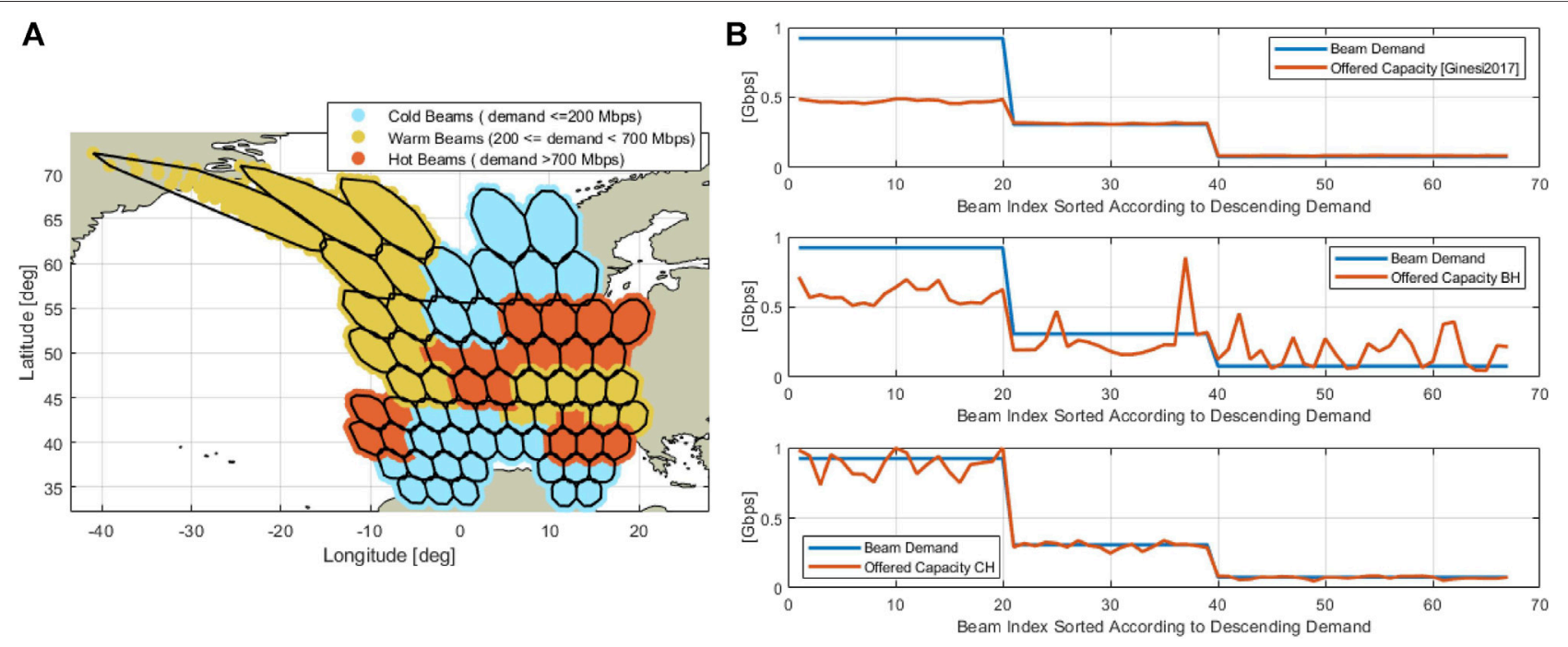

FIGURE 4 | Demand instance 1: (A) considered beam demand distribution; (B) offered per-beam capacity vs. per-beam demand.

widely used for resource allocation in satellite communications. The first corresponds to the amount of demanded capacity that cannot be satisfied with the actual offered capacity and is defined as $C_{\text {unmet }}=\sum_{i=1}^{N_{b}}\left(d_{i}-c_{i}\right)^{+}$, where $(x)^{+}=\max (0, x)$. The second corresponds to the amount of offered capacity which exceeds the demanded capacity, and it is given by $C_{\text {unused }}=\sum_{i=1}^{N_{b}}\left(c_{i}-d_{i}\right)^{+}$. Ideally, both unmet and unused capacity should be zero. 
TABLE 3 | Unmet and unused system capacity results for demand in Figure 4. Total demand is 26.46 Gbps.

\begin{tabular}{|c|c|c|c|c|c|}
\hline Technique & Illum. Ratio & $\begin{array}{l}\text { Offered capacity } \\
\text { (Gbps) }\end{array}$ & $\begin{array}{l}\text { Unmet capacity } \\
\text { (Gbps) }\end{array}$ & $\begin{array}{l}\text { Unused capacity } \\
\text { (Gbps) }\end{array}$ & Satisfaction \% (\%) \\
\hline Ginesi et al. (2017) & Not applicable & 17.75 & 9.03 & 0.32 & 85.40 \\
\hline \multirow[t]{3}{*}{ Conventional $\mathrm{BH}$} & $1 / 4$ & 27.17 & 8.99 & 9.71 & 79.89 \\
\hline & $1 / 6$ & 21.98 & 8.26 & 3.78 & 79.99 \\
\hline & $1 / 8$ & 16.64 & 9.83 & 0 & 64.61 \\
\hline \multirow[t]{3}{*}{ Proposed $\mathrm{CH}$} & $1 / 4$ & 30.95 & 2.58 & 7.07 & 93.66 \\
\hline & $1 / 6$ & 25.46 & 1.50 & 0.50 & 94.00 \\
\hline & $1 / 8$ & 18.89 & 7.57 & 0 & 72.79 \\
\hline
\end{tabular}

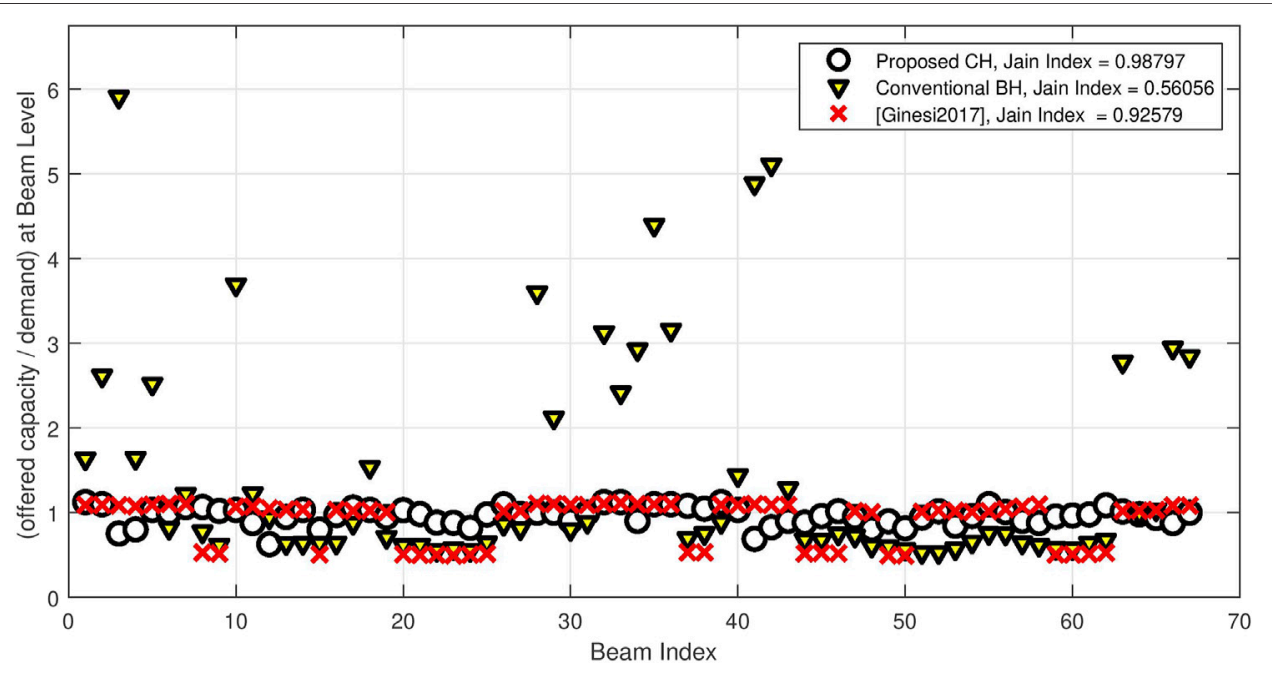

FIGURE $\mathbf{5}$ | Fairness results: per-beam offered capacity divided by per-beam demand and Jain's Fairness Index for demand instance shown in Figure 4.

Next, we present the performance evaluation of the proposed precoded $\mathrm{CH}$ system, which is compared with a conventional $\mathrm{BH}$ scheme and the study in Ginesi et al. (2017). The results presented herein have been obtained with the MATLAB-based software tool (SnT University of Luxembourg, 2020). For the conventional BH scheme, we use to solve the same problem as in (7) but assuming single beam clusters and, as a consequence, without precoding. All our results include the inter-cluster interference.

Figure 4A shows a particular demand distribution composed of three types of beams represented with different colors depending on their demand: high demand, medium demand, and low demand. Figure $\mathbf{4 B}$ shows the per-beam demand vs. the offered per-beam capacity for the three techniques under evaluation. The clustering option of four beams/clusters has been considered for the $\mathrm{CH}$ solution in this case. We can observe that Ginesi et al. (2017) satisfies well the low-demand beams while it struggles in meeting the demand of high-demand beams. Similarly, the conventional $\mathrm{BH}$ also shows difficulties in matching the demand of high-demand beams, while it shows some mismatch as well for the rest of the beams. Finally, the proposed $\mathrm{CH}$ is shown to properly follow the demand of any type of beam. Table 3 summarizes the system's unmet and unused capacity results, that is, $C_{\text {unmet }}$ and $C_{\text {unused }}$, for the demand in Figure 4. Table 3 also shows the total offered capacity and the satisfaction percentage, which represents the amount of beams that are satisfied. Note that the benchmark (Ginesi et al., 2017) does not apply a specific illumination ratio as the number of active beams per time slot change over time. The first observation is that the proposed $\mathrm{CH}$ technique with an illumination ratio of $1 / 6$ is providing the best unmet unused capacity trade-off, with both close to zero. Furthermore, $\mathrm{CH}$ is showing better demand satisfaction percentage too. The best results are achieved with an illumination ratio of $1 / 6$ because this provides an overall system offered capacity of $25.46 \mathrm{Gbps}$, which closely matches the overall requested demand of $26.46 \mathrm{Gbps}$. From the results in Table 3, we conclude that $\mathrm{CH}$ combined with a proper illumination ratio outperforms the benchmark schemes.

To complement the previous results, we now evaluate the fairness of the proposed solution in Figure 5, where the ratio of the per-beam demand vs. the achieved per-beam offered capacity is shown, as well as the resulting Jain's fairness index proposed in Jain et al. (1984). In this study, we use the Jain's fairness metric as a measure of how the offered capacity matches the demand at a beam level. For this, we define $\zeta_{i}$ as the ratio between the offered 

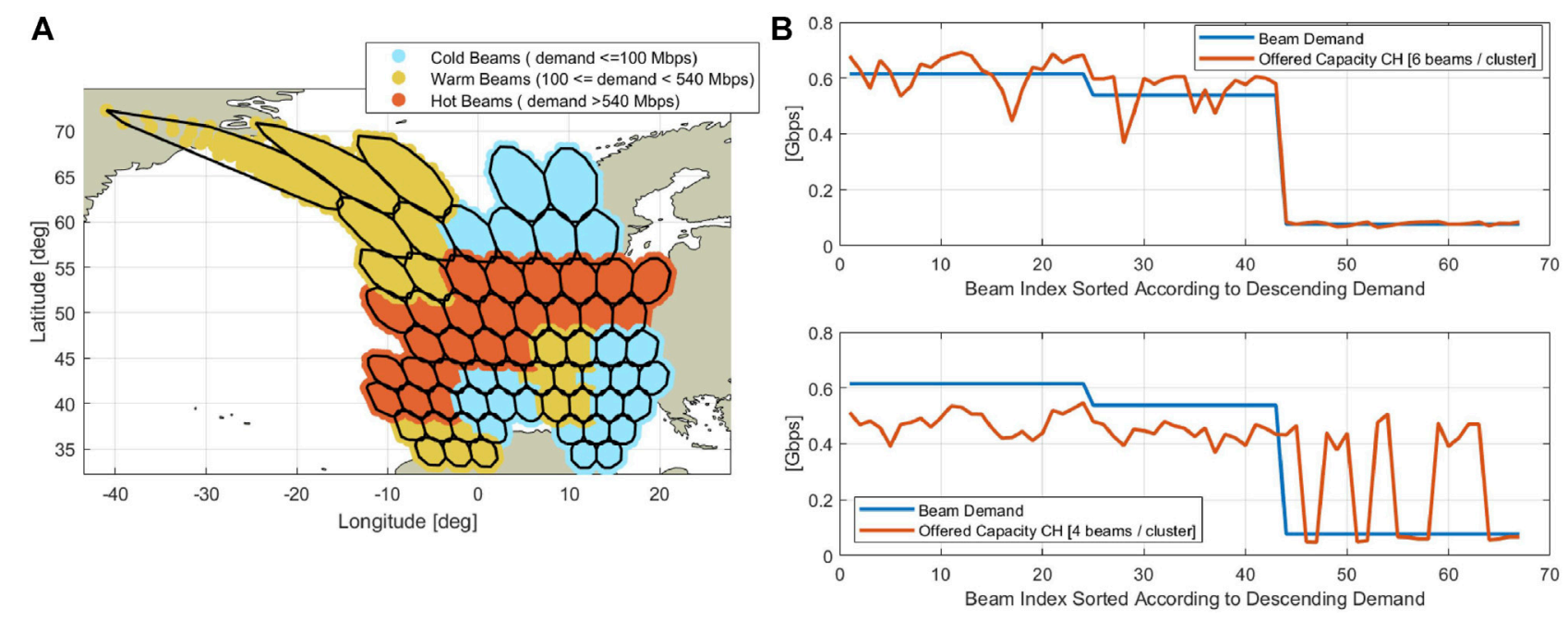

FIGURE 6 | Demand instance 2: (A) considered beam demand distribution; (B) offered per-beam capacity vs. per-beam demand.

TABLE 4 | Unmet and unused system capacity results for demand in Figure 6. Total demand is 26.85 Gbps.

\begin{tabular}{|c|c|c|c|c|c|}
\hline Technique & Illum. Ratio & $\begin{array}{l}\text { Offered capacity } \\
\text { (Gbps) }\end{array}$ & $\begin{array}{c}\text { Unmet capacity } \\
\text { (Gbps) }\end{array}$ & $\begin{array}{l}\text { Unused capacity } \\
\text { (Gbps) }\end{array}$ & Satisfaction (\%) \\
\hline Proposed CH & $1 / 4$ & 36.82 & 0.10 & 10.07 & 99.69 \\
\hline Six beams/cluster & $1 / 6$ & 27.58 & 0.86 & 1.59 & 97.08 \\
\hline Proposed CH & $1 / 4$ & 30.25 & 2.27 & 5.67 & 94.06 \\
\hline \multirow[t]{2}{*}{ Four beams/cluster } & $1 / 6$ & 25.90 & 5.38 & 4.44 & 82.58 \\
\hline & $1 / 8$ & 18.73 & 11.07 & 2.95 & 65.50 \\
\hline
\end{tabular}

capacity $C_{i}$ and the demanded/ideal capacity $D_{i}$, that is, $\zeta_{i}=\frac{C_{i}}{D_{i}}$, $i=1, \ldots, N_{b}$. In this context, the Jain's fairness index is defined as:

$$
J_{\mathrm{FI}}=\frac{\left(\sum_{i=1}^{N_{b}} \zeta_{i}\right)^{2}}{N_{b} \sum_{i=1}^{N_{b}} \zeta_{i}^{2}} \in\left[\frac{1}{N_{b}}, 1\right] .
$$

From Figure 5, it can be observed that the proposed $\mathrm{CH}$ outperforms the benchmark schemes in terms of fair per-beam demand satisfaction, as the values of $\zeta_{i}$ are closer to the idea value of 1 for all beams. The fairness of the proposed approach is confirmed by the Jain's index shown in the legend of Figure 5, where the proposed CH reaches a Jain's index of 0.99 (superior to that of the benchmarks).

Let us test now another demand instance with bigger demand areas, like the one shown in Figure 6A. For such big areas of demand, we expect the clustering of six beams/cluster to be a better fit. Table 4 shows the results achieved with the proposed $\mathrm{CH}$ for different clustering options and different illumination ratios. The best match is given by the six beam/cluster option with $1 / 6$ illumination ratio, where the unmet and unused capacity are equal to $0.86 \mathrm{Gbps}$ and $1.59 \mathrm{Gbps}$, respectively, with a satisfaction percentage of $97 \%$. The results shown in Table 4 provide evidence on the fact that not only it is important to select an accurate illumination ratio but also a clustering definition adapted to the expected demands. Finally, to confirm the superiority of the six beam/cluster for such type of demand distributions, Figure 6B provides the per-beam details of demand vs. offered capacity. It can be observe that the four beam/clustering option not only has problems in satisfying high-demands but also presents some mismatches for the low-demand beams.

\section{PRACTICAL CONSIDERATIONS}

\subsection{Channel State Information Acquisition}

Besides the synchronization aspects natural from beam-hopped transmission (Freedman et al., 2015), the main challenge of the proposed cluster hopping concept is the need of channel state information (CSI) at the gateway side. The most challenging problem in beam-hopped and precoded satellite systems is how often a ground terminal can measure its CSI vector (meaning the channel coefficient w.r.t. the satellite antennas). While the CSI estimation procedure can be based on already existing methods, the cluster hopping scheme requires some ad hoc adaptations due to time-variant nature of the cluster hopping procedure. In fact, since the set of illuminated beams changes over time, each user terminal is able to estimate a subset of coefficients of the complete CSI vector, which depends on the particular cluster than is being illuminated at that particular time instant. The latter can potentially lead to situations in which the gateway does not 
have all the needed coefficients to compute the precoding matrix. When the channel is relatively stable, the use of previous CSI estimates may solve the problem, otherwise joint processing of previous and new CSI coefficients would be required as well as prediction methods.

Furthermore, the general problem in beam-hopped satellite systems is how often a ground terminal can measure its CSI vector (meaning the channel coefficient w.r.t. the satellite antennas). Clearly, a ground terminal can only perform measurements when it is being illuminated, and the number of measured CSI components depends on the particular cluster than it is being illuminated at that particular time instant. We should distinguished two cases:

- Illumination pattern composed of nonoverlapping clusters: this is the case assumed in this study. In this case, each ground terminal only needs the knowledge of the CSI components related to the satellite antennas that are active in the cluster that it belongs to. Therefore, we propose to rely on the CSI gathered in the previous time instant that this particular cluster was illuminated (which of course will imply some additional delay depending on the illumination period).

- Illumination pattern composed of overlapping clusters: this would be the case when dealing with high traffic demand areas that need to be illuminateds most of the time. For the sake of clarity, let us assume an example composed of three beams and two non-orthogonal clusters, the first cluster composed of beam 1 and beam 2, and the second cluster composed of beam 2 and beam 3. Let us focus on the terminals belonging to the beam 2 coverage area. Note that beam 2 is always active but once together with beam 1 and once together with beam 3. Therefore, this configuration implies that high-demand beams that are more often illuminated (i.e., beam 2) will have more accurate CSI that low-demand beams (i.e., beam 1 and beam 3), which are less often activated.

Generally speaking, we do not foresee the outdated CSI to have a strong impact. This is because a single DVB-S2(X) super-frame is enough to obtain a good channel estimation and, therefore, the outdated CSI will only be needed for the initial (single) superframe.

\subsection{Future Payload Antenna Systems}

The results presented in this study have been obtained assuming a Direct Radiating Array (DRA)-based footprint pattern, which have been generated with internal software by ESA in $20 \mathrm{GHz}$, with 750 elements spaced five lambda, able to provide 67 beams within the desired coverage area. The trends in the satellite communications industry are evolving towards more advanced antenna architectures, for example, (defocused) phased array fed reflector (PAFR), whose phase response may differ from conventional single-feed-per-beam architecture or the DRA considered in this study. The PAFR may offer some benefits such as lower cost, high beam resolution, and smaller array size.

\section{CONCLUSIONS AND FUTURE RESEARCH DIRECTIONS}

In this study, we have proposed the combination of precoding and time-flexible payloads with $\mathrm{BH}$ capabilities. Focusing on the convergence of both techniques, we have proposed the so-called cluster hopping $(\mathrm{CH})$ concept, which seamlessly combines these two paradigms and utilizes the strong points of each one. Supporting results based on numerical simulations are provided which validate the effectiveness of the proposed system in comparison with conventional $\mathrm{BH}$ and other works available in the literature. Particularly, $\mathrm{CH}$ shows great promise when dealing with high demands that cover large portionals of the Earth, thus spanning multiple satellite beams. The results of this study have highlighted the importance of an appropriate clustering design together with an appropriate illumination ratio, both based on the expected demand distribution. The latter opens opportunities for future research in this subject, namely the optimal clustering definition based on demand distribution input and the appropiate portion of beams that needs to be activated at a time. Furthermore, this study has considered the transmit power out of the scope for the sake of clarity but the transmit power represents another degree of freedom that can be considered within the optimization problem.

\section{DATA AVAILABILITY STATEMENT}

The original contributions presented in the study are included in the article/Supplementary Material; further inquiries can be directed to the corresponding author.

\section{AUTHOR CONTRIBUTIONS}

EL led this manuscript in both technical contribution, production of results, and writing. MK contributed to the technical ideas and help in producing the numerical results. HA-H and NM contributed to the developed techniques. SC contributed in conceiving the main idea and supervised the findings of this study. All authors contributed to the manuscript and approved the submitted version.

\section{FUNDING}

This study has been partially supported by the Luxembourg National Research Fund (FNR) under the project FlexSAT (C19/IS/13696663) and by the European Space Agency (ESA) under the activity FlexPreDem: Demonstrator of Precoding Techniques for Flexible Broadband Systems. Please note that the views of the authors of this study do not necessarily reflect the views of ESA.

\section{ACKNOWLEDGMENTS}

The authors would like to thank Stefano Andrenacci and Joel Grotz from SES and Daniel P. Arapoglou from ESA for their support and valuable discussions and suggestions during the executions of this work. 


\section{REFERENCES}

AIRBUS (2021). Flexible Payloads. Available at: : https://www.airbus.com/space/ telecommunications-satellites/flexible-payloads.html (Accessed May 24, 2021).

Alegre-Godoy, R., Alagha, N., and Vázquez-Castro, M. A. (2012). "Offered Capacity Optimization Mechanisms for Multi-Beam Satellite Systems," in 2012 IEEE International Conference on Communications (ICC) (IEEE), 3180-3184. doi:10.1109/ICC.2012.6364414

Angeletti, P., Prim, D. F., and Rinaldo, R. (2012). "Beam Hopping in Multi-Beam Broadband Satellite Systems: System Performance and Payload Architecture Analysis," in 24th AIAA International Communications Satellite Systems Conference (San Diego, California: AIAA). doi:10.2514/6.2006-5376

Anzalchi, J., Couchman, A., Gabellini, P., Gallinaro, G., D'Agristina, L., Alagha, N., et al. (2010). "Beam Hopping in Multi-Beam Broadband Satellite Systems: System Simulation and Performance Comparison with Non-hopped Systems," in Adv. Satellite Multimedia Systems Conf./Signal Process. For Space Commun. Workshop (Cagliari, Italy: ASMS/SPSC). doi:10.1109/ASMS-SPSC.2010.5586860

Arapoglou, P.-D., Ginesi, A., Cioni, S., Erl, S., Clazzer, F., Andrenacci, S., et al. (2016). Dvb-s2x-enabled Precoding for High Throughput Satellite Systems. Int. J. Satell. Commun. Netw. 34, 439-455. doi:10.1002/sat.1122

Cocco, G., de Cola, T., Angelone, M., Katona, Z., and Erl, S. (2018). Radio Resource Management Optimization of Flexible Satellite Payloads for DVB-S2 Systems. IEEE Trans. Broadcast. 64, 266-280. doi:10.1109/TBC.2017.2755263

Cola, T. D., Tarchi, D., and Vanelli-Coralli, A. (2015). Future Trends in Broadband Satellite Communications: Information Centric Networks and Enabling Technologies. Int. J. Satellite Commun. Networking 33, 5. doi:10.1002/sat.1101

DVB-S2X (2014). Second Generation Framing Structure, Channel Coding and Modulation Systems for Broadcasting, Interactive Services, News Gathering and Other Broadband Satellite Applications; Part 2: DVB-S2 Extensions (DVBS2x), Document ETSI EN 302 307-2. Available at: https://www.etsi.org/deliver/ etsi_en/302300_302399/30230702/01.01.01_20/en_30230702v010101a.pdf.

ESA project BEAMHOP (2016). Beam Hopping Techniques in Multi-Beam Satellite Systems. Noordwijk, Netherlands: European Space Agency. Available at: https:// artes.esa.int/projects/beam-hopping-techniques-multi-beam-satellite-systemseads-astrium.

ESA Project FlexPreDem (2020). Demonstrator of Precoding Techniques for Flexible Broadband Satellite Systems. Available at: https://artes.esa.int/projects/flexpredem.

ESA project LiveSatPreDem (2020). Live Satellite Precoding Demonstration. Available at: https://artes.esa.int/projects/livesatpredem.

Freedman, A., Rainish, D., and Gat, Y. (2015). "Beam Hopping - How to Make it Possible," in Proc. Of Ka and Broadband Communication Conference (IEEE).

Ginesi, A., Re, E., and Arapoglou, P. (2017). "Joint Beam Hopping and Precoding in HTS Systems," in 9th Int. Conf. On Wireless and Satellite Systems (WiSATS) (IEEE).

Guidotti, A., and Vanelli-Coralli, A. (2020). Clustering Strategies for Multicast Precoding in Multibeam Satellite Systems. Int. J. Satell Commun. Netw. 38, 85-104. doi:10.1002/sat.1312

GUROBI (2021). Mathematical Programming Solver. Available at: https://www. gurobi.com/.

Honnaiah, P. J., Lagunas, E., Spano, D., Maturo, N., and Chatzinotas, S. (2021). "Demand-based Scheduling for Precoded Multibeam High-Throughput Satellite Systems," in IEEE Wireless Communications and Networking Conference (WCNC) (IEEE). doi:10.1109/wcnc49053.2021.9417300

Jain, R., Chiu, D., and Hawe, W. (1984). A Quantitative Measure of Fairness and Discrimination for Resource Allocation in Shared Computer System. Hudson, MA: DEC Technical Report, 301.
Kibria, M. G., Lagunas, E., Maturo, N., Spano, D., and Chatzinotas, S. (2019) "Precoded Cluster Hopping in Multi-Beam High Throughput Satellite Systems," in 2019 IEEE Global Communications Conference (GLOBECOM) (IEEE), 1-6. doi:10.1109/globecom38437.2019.9013589

Kisseleff, S., Lagunas, E., Abdu, T., Chatzinotas, S., and Ottersten, B. (2020). Radio Resource Management Techniques for Multibeam Satellite Systems. IEEE Commun. Lett. 25, 2448-2452. doi:10.1109/ LCOMM.2020.3033357

Kodheli, O., Lagunas, E., Maturo, N., Sharma, S. K., Shankar, B., Mendoza, J. F., et al. (2020). "Satellite Communications in the New Space Era: A Survey and Future Challenges," in IEEE Communications Surveys Tutorials (ArXiv: 2002.08811).

Kyrgiazos, A., Evans, B. G., and Thompson, P. (2014). On the Gateway Diversity for High Throughput Broadband Satellite Systems. IEEE Trans. Wireless Commun. 13, 5411-5426. doi:10.1109/TWC.2014.2339217

Lei, L., Lagunas, E., Yuan, Y., Kibria, M. G., Chatzinotas, S., and Ottersten, B. (2020). Beam Illumination Pattern Design in Satellite Networks: Learning and Optimization for Efficient Beam Hopping. IEEE Access 8, 136655-136667. doi:10.1109/access.2020.3011746

NetWorld 2020 (2019). White Paper: SatCom Resources for Smart andSustainable Networks and Services. Available at: https://www.networldeurope.eu/ whitepaper-satcom-resources-for-smart-and-sustainable-networks-and-services/ (Accessed May 24, 2021).

SES (2020). Unlocking Agility of SES Satellites with Adaptive Resource Control. Available at: https://www.ses.com/blog/unlocking-agility-ses-satellites-adaptiveresource-control.

SnT University of Luxembourg (2020). Demonstrator of Precoding Techniques for Flexible Broadband Satellite Systems. Available at: https://wwwfr.uni.lu/snt/ research/sigcom/sw_simulators/flexpredem.

Vazquez, M. A., Perez-Neira, A., Christopoulos, D., Chatzinotas, S., Ottersten, B., Arapoglou, P.-D., et al. (2016). Precoding in Multibeam Satellite Communications: Present and Future Challenges. IEEE Wireless Commun. 23, 88-95. doi:10.1109/mwc.2016.1500047wc

ViaSat Inc (2018). Satellite Internet Services. Available at: https://www. satelliteinternet.com/ (Accessed May 24, 2021).

Zhang, F., Zuo, Y., and Sun, H. (2017). "Techniques of Rain Fade Countermeasures in Ka-Band Satellite Communication on Ships," in 2017 16th International Conference on Optical Communications and Networks (ICOCN) (IEEE), 1-3. doi:10.1109/icocn.2017.8121382

Conflict of Interest: The authors declare that the research was conducted in the absence of any commercial or financial relationships that could be construed as a potential conflict of interest.

Publisher's Note: All claims expressed in this article are solely those of the authors and do not necessarily represent those of their affiliated organizations, or those of the publisher, the editors, and the reviewers. Any product that may be evaluated in this article, or claim that may be made by its manufacturer, is not guaranteed or endorsed by the publisher.

Copyright $\odot 2021$ Lagunas, Kibria, Al-Hraishawi, Maturo and Chatzinotas. This is an open-access article distributed under the terms of the Creative Commons Attribution License (CC BY). The use, distribution or reproduction in other forums is permitted, provided the original author(s) and the copyright owner(s) are credited and that the original publication in this journal is cited, in accordance with accepted academic practice. No use, distribution or reproduction is permitted which does not comply with these terms. 